\title{
PRECISION GRAVITY TESTS BY ATOM INTERFEROMETRY
}

\author{
G. M. TINO*, A. ALBERTI, A. BERTOLDI, L. CACCIAPUOTI ${ }^{\dagger}$, \\ M. DE ANGELIS ${ }^{\ddagger}$, G. FERRARI, A. GIORGINI, V. IVANOV, \\ G. LAMPORESI, N. POLI, M. PREVEDELLI ${ }^{\S}$, F. SORRENTINO \\ Dipartimento di Fisica and LENS Laboratory - Università di Firenze \\ Istituto Nazionale di Fisica Nucleare, Sezione di Firenze \\ via Sansone 1, Polo Scientifico, I-50019 Sesto Fiorentino (Firenze), Italy
}

\begin{abstract}
We report on experiments based on atom interferometry to determine the gravitational constant $G$ and test the Newtonian gravitational law at micrometric distances. Ongoing projects to develop transportable atom interferometers for applications in geophysics and in space are also presented.
\end{abstract}

\section{Introduction}

Advances in atom interferometry led to the development of new methods for fundamental physics experiments and for applications. In particular, atom interferometers are new tools for experimental gravitation as, for example, for precision measurements of gravity acceleration [1] and gravity gradients [2], for the determination of the Newtonian constant $G[3,4]$, for testing general relativity $[5,6]$ and $1 / r^{2}$ law $[7-10]$, and for possible applications in geophysics. Ongoing studies show that future experiments in space will allow to take full advantage of the potential sensitivity of atom interferometers [11]. The possibility of using atom interferometry for gravitational waves detection was also investigated (see [12] and references therein).

In this paper, we report on experiments we are performing using atom interferometry to determine $G$ and test the Newtonian gravitational law at

\footnotetext{
*E-mail: guglielmo.tino@fi.infn.it - Web: www.lens.unifi.it/tino

${ }^{\dagger}$ Permanent address: ESA Research and Scientific Support Department, ESTEC, Keplerlaan 1- P.O. Box 299, 2200 AG Nordwijk ZH, The Netherlands

$\ddagger$ On leave from: Istituto Cibernetica CNR, 80078 Pozzuoli (Napoli), Italy

${ }_{\text {}}$ Permanent address: Dipartimento di Chimica Fisica, Università di Bologna, Via del Risorgimento 4, 40136 Bologna, Italy
} 
micrometric distances. We also present ongoing projects to develop transportable atom interferometers for applications in geophysics and in space.

\section{Determination of $G$ by atom interferometry}

The Newtonian constant of gravity $G$ is one of the most measured fundamental physical constants and at the same time the least precisely known. The extreme weakness of the gravitational interaction and the impossibility of shielding the effects of gravity make it difficult to measure $G$, while keeping systematic effects well under control. Despite the numerous experiments, the uncertainty on $G$ has improved only by one order of magnitude in the last century [13]. Many of the experiments performed to date are based on the traditional torsion pendulum method, direct derivation of the historical experiment performed by Cavendish in 1798. Recently, many groups have set up new experiments based on different concepts and with completely different systematics. However, the most precise measurements available today still show substantial discrepancies, limiting the accuracy of the 2006 CODATA recommended value for $G$ to 1 part in $10^{4}$. From this point of view, the realization of conceptually different experiments can help to identify still hidden systematic effects and therefore improve the confidence in the final result.

We use atom interferometry to perform precision measurements of the differential acceleration experienced by two samples of laser-cooled rubidium atoms under the influence of nearby tungsten masses. In our experiment, specific efforts have been devoted to the control of systematic effects related to atomic trajectories, positioning of source masses, and stray fields. In particular, the high density of tungsten and the distribution of the source masses are crucial in our experiment to compensate for the Earth gravity gradient and reduce the sensitivity of the measurement to the initial position and velocity of the atoms. The measurement, repeated for two different configurations of the source masses, is modeled by a numerical simulation which takes into account the mass distribution and the evolution of atomic trajectories. The comparison of measured and simulated data provides the value of the Newtonian gravitational constant $G$. Proof-of-principle experiments with similar schemes using lead masses were already presented in $[3,4]$. In our interferometer, laser pulses are used to stimulate ${ }^{87} \mathrm{Rb}$ atoms on the two-photon Raman transition between the hyperfine levels $F=1$ and $F=2$ of the ground state [14]. The light field is generated by two counter-propagating laser beams with wave vectors $\mathbf{k}_{1}$ and $\mathbf{k}_{2} \simeq-\mathbf{k}_{1}$ aligned along the vertical direction. The laser frequencies $\nu_{1}$ 
and $\nu_{2}$ match the resonance condition $\nu_{1}-\nu_{2}=\nu_{0}$, where $h \nu_{0}$ is the energy associated to the $F=1 \rightarrow F=2$ transition. The atom interferometer, obtained with a $\pi / 2-\pi-\pi / 2$ sequence of Raman pulses, drives the atoms on two independent paths along which the quantum mechanical phases of the atomic wavepackets independently evolve. In the presence of a gravity field, atoms experience a phase shift $\Phi=\mathbf{k} \cdot \mathbf{g} T^{2}$, where $\mathbf{k}=\mathbf{k} 1-\mathbf{k}_{2}, 2 T$ is the duration of the pulse sequence, and $\mathbf{g}$ is gravity acceleration. A measurement of the phase $\Phi$ is equivalent to an acceleration measurement. The gravity gradiometer consists of two absolute accelerometers operated in differential mode. Two spatially separated atomic clouds aligned along the vertical direction are simultaneously interrogated by the same $\pi / 2-\pi-\pi / 2$ pulse sequence. The difference of the phase shifts detected on each interferometer provides a direct measurement of the differential acceleration induced by gravity on the two atomic samples. This method has the major advantage of being highly insensitive to noise sources appearing in common mode on both interferometers. In particular, any spurious acceleration induced by vibrations or seismic noise on the common reference frame identified by the vertical Raman beams is efficiently rejected by the differential measurement technique.

Figure 1 shows a schematic of the experiment. The gravity gradiometer set-up and the configurations of the source masses $\left(C_{1}\right.$ and $\left.C_{2}\right)$ used for the $G$ measurement are visible. The atom interferometer apparatus and
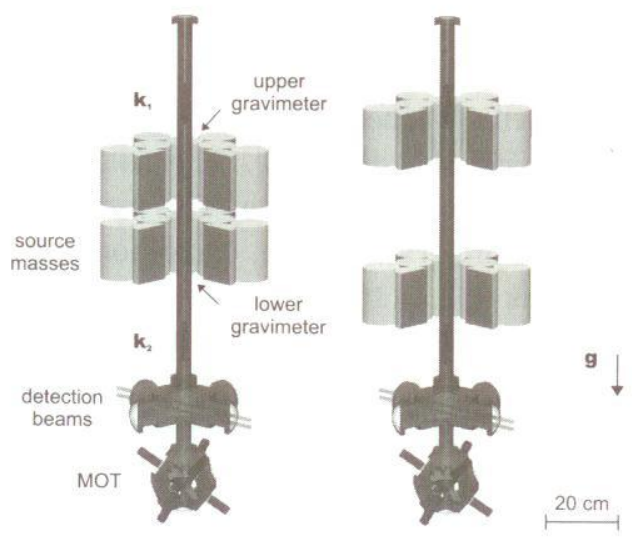

Fig. 1. Schematic of the experiment showing the gravity gradiometer set-up with the Raman beams propagating along the vertical direction. For the measurement of $G$, the position of the source masses is alternated between configuration $C_{1}$ (left) and $C_{2}$ (right). 
the source masses assembly are described in detail elsewhere [3,15]. In the vacuum chamber at the bottom of the apparatus, a magneto-optical trap (MOT) collects rubidium atoms from the vapor produced by getters. After turning the MOT magnetic field off, the atomic sample is launched vertically along the symmetry axis of the vacuum tube by using the moving molasses technique. The gravity gradient is probed by two atomic clouds moving in free flight along the vertical axis of the apparatus and simultaneously reaching the apogees of their ballistic trajectories at $60 \mathrm{~cm}$ and $90 \mathrm{~cm}$ above the MOT. Such a geometry, requiring the preparation and the launch of two samples with high atom numbers in a time interval of about $100 \mathrm{~ms}$, is achieved by juggling the atoms loaded in the MOT. The interferometers are realized at the center of the magnetically shielded vertical tube shown in Fig. 1. The three-pulse interferometer has a duration of $2 T=320 \mathrm{~ms}$. The $\pi$ pulse lasts $48 \mu$ s and occurs $5 \mathrm{~ms}$ after the atomic clouds reach their apogees. In this configuration, only one pair of counterpropagating laser beams with frequencies $\nu_{1}$ and $\nu_{2}$ and crossed linear polarizations is able to stimulate the atoms on the two-photon transition. At the end of their ballistic flight, the population of the ground state is measured by selectively exciting the atoms in both hyperfine levels of the ground state and detecting the light-induced fluorescence emission. We typically detect $10^{5}$ atoms on each rubidium sample at the end of the interferometer sequence. Even if the phase noise induced by vibrations washes out the atom interference fringes, the signals simultaneously detected on the upper and lower accelerometer remain coupled and preserve a fixed phase relation. Therefore, when the trace of the upper accelerometer is plotted as a function of the lower one, experimental points distribute along an ellipse. The differential phase shift is then obtained from the eccentricity and the rotation angle of the ellipse fitting the data [16]. The Allan deviation shows the typical behavior expected for white noise. The instrument has a sensitivity of $140 \mathrm{mrad}$ at $1 \mathrm{~s}$ of integration time, corresponding to a sensitivity to differential accelerations of $3.5 \cdot 10^{-8} \mathrm{~g}$ in $1 \mathrm{~s}$.

The source masses [15] are composed of 24 tungsten alloy (INERMET IT180) cylinders, for a total mass of about $516 \mathrm{~kg}$. They are positioned on two titanium platforms and distributed in hexagonal symmetry around the vertical axis of the tube (see Fig. 1).

The value of $G$ was determined from a series of gravity gradient measurements performed by periodically changing the vertical position of the source masses between configuration $C_{1}$ and $C_{2}$ while keeping the atomic trajectories fixed. Because of the high density of tungsten, the gravitational 
field produced by the source masses is able to compensate for the Earth gravity gradient. As a consequence, the acceleration becomes less sensitive to the positions of the atomic clouds around extremal points, allowing for a better control of systematic effects and a relaxation of the requirements on the knowledge of atomic trajectories.

Figure 2 shows a data sequence used for the measurement of $G$. Each phase measurement is obtained by fitting a 24-point scan of the atom interference fringes to an ellipse. After an analysis of the error sources affecting our measurement, we obtain a value of $G=6.667 \cdot 10^{-11} \mathrm{~m}^{3} \mathrm{~kg}^{-1} \mathrm{~s}^{-2}$, with a statistical uncertainty of $\pm 0.011 \cdot 10^{-11} \mathrm{~m}^{3} \mathrm{~kg}^{-1} \mathrm{~s}^{-2}$ and a systematic uncertainty of $\pm 0.003 \cdot 10^{-11} \mathrm{~m}^{3} \mathrm{~kg}^{-1} \mathrm{~s}^{-2}$. Our measurement is consistent with the 2006 CODATA value within one standard deviation. The main contribution to the systematic error on the $G$ measurement derives from the positioning accuracy of the source masses. This error will be reduced by about one order of magnitude by measuring the position of the tungsten cylinders with a laser tracker. Eventually, uncertainties below the $10 \mathrm{ppm}$ level could be reached with this scheme using for the source mass a material with a higher density homogeneity.
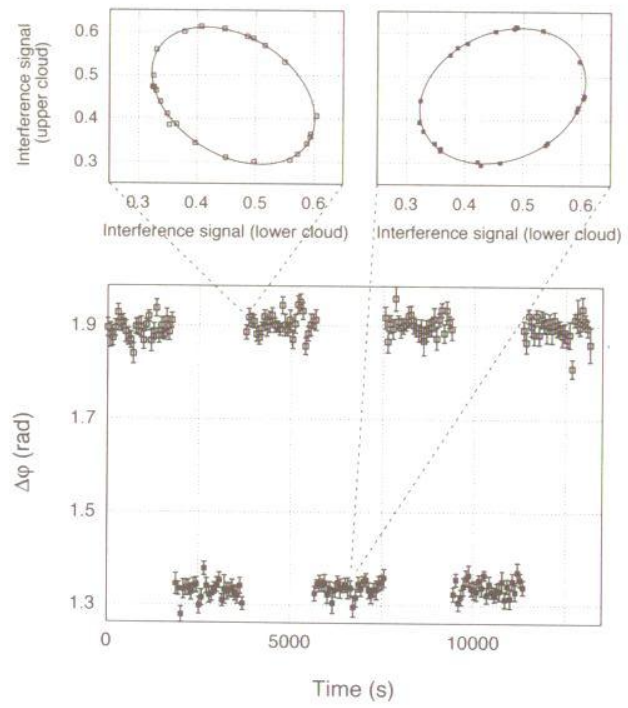

Fig. 2. Typical data set showing the modulation of the differential phase shift measured by the atomic gravity gradiometer when the distribution of the source masses is alternated between configuration $C_{1}$ and $C_{2}$. Each phase measurement is obtained by fitting a 24-point scan of the atom interference fringes to an ellipse. 


\section{Precision gravity measurements at $\mu m$ scale with laser-cooled $\mathrm{Sr}$ atoms in an optical lattice}

The extremely small size of atomic sensors can lead to applications for precision measurements of forces at micrometer scale. The investigation of forces at small length scales is indeed a challenge for present research in physics for the study of surfaces, of the Casimir effect, and in the search for deviations from Newtonian gravity predicted by recent theories beyond the standard model.

We showed [10] that using laser-cooled ${ }^{88} \mathrm{Sr}$ atoms in an optical lattice, persistent Bloch oscillations can be observed for a time $\sim 10 \mathrm{~s}$, because of remarkable properties of immunity of this atom from perturbations due to stray fields and interatomic collisions. This system can reach an unprecedented sensitivity as sensor to measure gravity acceleration on micrometer scale with ppm precision opening the way to the investigation of small-scale gravitational forces in regions so far unexplored.

The experiment starts with trapping and cooling $\sim 5 \times 10^{7}{ }^{88} \mathrm{Sr}$ atoms at $3 \mathrm{mK}$ in a magneto-optical trap (MOT) operating on the ${ }^{1} \mathrm{~S}_{0^{-}}{ }^{1} \mathrm{P}_{1}$ blue resonance line at $461 \mathrm{~nm}$. The temperature is then further reduced by a second cooling stage in a red MOT operating on the ${ }^{1} \mathrm{~S}_{0^{-}}{ }^{3} \mathrm{P}_{1}$ narrow transition at $689 \mathrm{~nm}$ and finally we obtain $\sim 5 \times 10^{5}$ atoms at $400 \mathrm{nK}$. After this preparation phase, the red MOT is switched off and a one-dimensional optical lattice is switched on adiabatically in $50 \mu$ s. The lattice potential

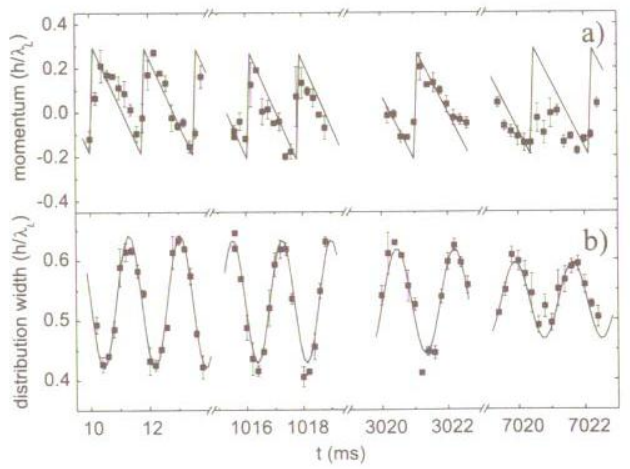

Fig. 3. Bloch oscillation of ${ }^{88} \mathrm{Sr}$, atoms in the vertical 1-dimensional optical lattice under the effect of gravity. Two quantities are extracted from the analysis of the data: The vertical momentum of the oscillating atoms (a) and the width of the atomic momentum distribution (b). 
is originated by a single-mode frequency-doubled Nd:YVO4 laser $\left(\lambda_{L}=\right.$ $532 \mathrm{~nm}$ ). The beam is vertically aligned and retro-reflected by a mirror producing a standing wave with a period $\lambda_{L} / 2=266 \mathrm{~nm}$. We obtain a diskshaped sample of $10^{5}$ atoms at $T \sim 400 \mathrm{nK}$ with a vertical rms width of $\sim$ $12 \mu \mathrm{m}$ and a horizontal radius of $\sim 150 \mu \mathrm{m}$. We observe Bloch oscillations in the vertical atomic momentum by releasing the optical lattice at a variable delay, and by imaging the atomic distribution after a fixed time of free fall. Figure 3 shows the signal recorded for $7 \mathrm{~s}$, corresponding to $\sim 8000$ oscillations. The coherence time for the Bloch oscillation is $\sim 12 \mathrm{~s}$. These values are the highest ever observed for Bloch oscillations in atomic systems. Measuring the oscillation frequency we determine the vertical force on the atoms, that is, Earth gravity with a resolution of $5 \times 10^{-6}$.

In the effort to increase the sensitivity, recently we investigated strontium atoms trapped in phase-modulated optical lattices. We found that we can induce a broadening of the atomic distribution in the lattice potential with a phase modulation of the lattice at frequencies multiple of Bloch frequency. We observed a resonant broadening up to the 5th harmonic which corresponds to a hop through 5 lattice sites (Fig. 4). All the resonance spectra exhibit a Fourier-limited width for excitation times as long as $2 \mathrm{~s}$. The resulting high-resolution measurement of Wannier-Stark levels of the atomic wavefunction in the gravity potential allows to determine the local gravity acceleration with a relative precision $\sim 10^{-6}$.

When studying atom-surface interactions, one key point is the precision of sample positioning close to the surface. In our experiment, the optical lattice is also used for an accurate positioning of the sample close to the

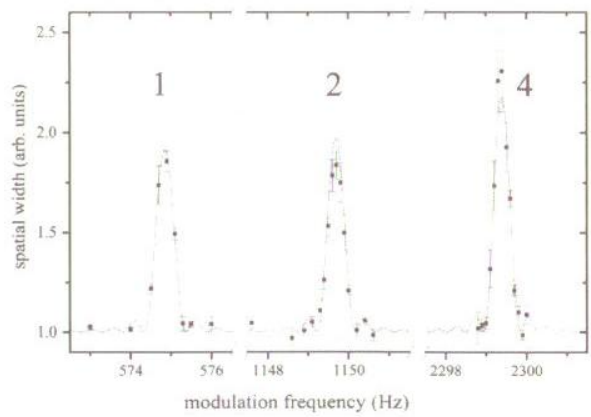

Fig. 4. Spatial width of the atomic distribution for $2 \mathrm{~s}$ phase modulation of the optical lattice at the 1st, 2nd and 4th harmonics of the Bloch frequency. 
surface. We translate the atomic sample along the lattice axis by applying a relative frequency offset to the counterpropagating laser beams producing the lattice. In this way, we place the atoms close to a transparent test surface placed $\sim 45 \mathrm{~mm}$ far from the MOT. We measure the atoms number and the phase of the Bloch oscillation with absorption imaging after bringing the atoms back to the original position. In Fig. 5.a), we show the number of atoms recorded after an elevator round-trip, as a function of the travelled distance. A sudden drop, corresponding to the loss of atoms hitting the test surface, is clearly visible. The plot provides a measurement of the vertical size of the atomic sample. This scheme directly applies to transparent materials. In order to study metallic surfaces, the atomic sample in the optical lattice is displaced by means of optical components mounted on micrometric translation stages. In that case, the optical lattice is produced by retroreflecting the laser beam on the test surface itself. The minimum attainable atom-surface distance is limited by the vertical size of the atomic distribution. For experiments at distances below $10 \mu \mathrm{m}$, we compress our sample using an optical tweezer. This is obtained with a strongly astigmatic laser beam with the vertical focus centered on the atoms. Figure 5.b) shows an image of the atoms trapped in the optical tweezer.

Deviations from the Newtonian gravity law are usually described assuming a Yukawa-type potential with two parameters, $\alpha$ giving the relative strength of any new effect compared to Newtonian gravity and $\lambda$ its range. Experiments searching for possible deviations have set bounds for the parameters $\alpha$ and $\lambda$. Recent results using microcantilever detectors lead to extrapolated limits $\alpha \sim 10^{4}$ for $\lambda \sim 10 \mu \mathrm{m}[17,18]$. The small size and high sensitivity of the atomic probe allow a direct, model-independent measure-
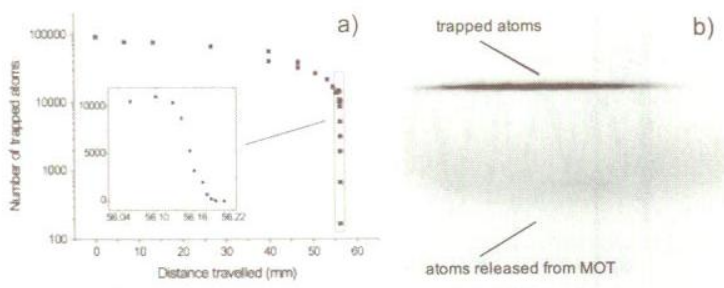

Fig. 5. a) Number of atoms in the lattice versus vertical displacement. The inset shows the region close to the test surface. The vertical displacement is varied by changing the duration of the motion at uniform velocity. b) In-situ absorption image of the atomic sample trapped in the optical tweezer. Untrapped falling atoms are also visible. 
ment at distances of a few $\mu \mathrm{m}$ from the source mass with no need for modeling and extrapolation as in the case of macroscopic probes. This allows to directly access unexplored regions in the $\alpha-\lambda$ plane. Also, in this case quantum objects are used to investigate gravitational interaction. If we consider a thin layer of a material of density $\rho$ and thickness $d$, the Newtonian gravitational acceleration due to the source mass is $a=2 \pi G \rho d$; for $d \sim 10 \mu \mathrm{m}$ and $\rho \simeq 20 \mathrm{~g} / \mathrm{cm}^{3}$, as for gold or tungsten, the resulting acceleration is $a \sim 10^{-10} \mathrm{~ms}^{-2}$. Measuring $\nu_{B}$ at a distance of $10 \mu \mathrm{m}$ from the surface will then provide a direct test of present constraints on $\alpha$ [18]. For smaller distances, around $5 \mu \mathrm{m}$, it is possible to improve present limits on $\alpha$ by more than two orders of magnitude in the corresponding $\lambda$ range. Even shorter distances could probably be accessed, also considering a related scheme based on a Sr lattice clock [19].

Non-gravitational effects (Van der Waals, Casimir forces), also present in other experiments, can be reduced by using a conductive screen and performing differential measurements with different source masses placed behind it. For this experiment we developed a source mass made of a sandwich of $\mathrm{Al}$ and $\mathrm{Au}$ layers covered by an $\mathrm{Au}$ layer. This layer acts as the mirror to produce the optical standing wave and as the conductive screen. Placing the atoms in proximity of the different materials with different mass densities, mass-dependent effects can be investigated. Also, by performing the experiment with different isotopes of $\mathrm{Sr}$, having different masses but the same electronic structure, gravitational forces can be distinguished from other surface interactions.

\section{Transportable atom gravimeters for geophysics applications and future experiments in space}

Inertial and rotational sensors using atom interferometry display a potential for replacing other state-of-the-art sensors for e.g. geophysics and space applications. The intrinsic benefits making direct use of fundamental quantum processes promise significant advances in performance, usability, and efficiency, from the deployment of highly optimized devices on satellites in space or from the use of ground based transportable devices.

\subsection{Geophysics applications}

The knwoledge of the value of $\mathrm{g}$ and its time and space variations is of interest to a wide field of physical sciences connected to geophysics and geodesy. Surface gravity measurements on local scale can help in under- 
standing active tectonics areas dynamics, faulting during the interseismic phase, fluid migration/diffusion due to stress changes. A variety of maninduced physical and chemical processes are known to produce substantial vertical displacements of the Earth's surface and are related to mass or fluid extraction or to subsurface pore fluid flows. Gravity measurements are used in acquifier and reservoirs monitoring, monitoring of fluid infiltration and water table rise near nuclear repositories and monitoring of subsidence and mining effects. Recently, micro-gravimetric observations have found an important field of application in volcanoes monitoring. Variations in the local gravity field were observed prior and during eruptive episodes of variable size at a number of volcanoes worldwide. It appears that crucial mass redistribution in geodynamics occur over time scales spanning the $1-10^{6} \mathrm{~s}$ interval and have amplitudes ranging from 10 microgals up to hundreds of microgals $\left(1 \mathrm{Gal}=1 \mathrm{~cm} / \mathrm{s}^{2}\right)$. Some of these phenomena are observed very early and occur before other phenomena as strain deformations or seismic signals. These considerations indicate that the continuous observation of the local gravity field using sensitive instrumentation with comparable accuracy on long periods is a major goal to be attained towards a better understanding of active volcanic systems and prediction of eruptive activity.

Prototypes of transportable gravimeters based on atom interferometry have been realized by different labs. In Stanford, a transportable system was developed and used to measure the components of the gravity tensor. At JPL, a compact gravity gradiometer is being developed. A transportable gravimeter based on atom interferometry was developed for metrological applications at SYRTE in Paris.

Our group is involved in these activities in the frame of a European STREP/NEST project (FINAQS) and with support from the Istituto Nazionale di Geofisica e Vulcanologia (INGV). We are developing a transportable atomic gravimeter that will be used for geophysics applications. Our main interest is in vulcanoes monitoring to investigate the possibility of predicting eruptions. The high sensitivity and long term stability achievable with an atom gravimeter are important characteristics for this application.

\subsection{Space applications}

Future experiments in space will allow to take full advantage of the potential sensitivity of cold atom interferometers as acceleration or rotation sensors. Indeed, atomic quantum sensors can reach their ultimate performances if operated in space because of the extremely long achievable interaction time and the vibration-free environment. 
In Europe, the interest on application of atomic quantum sensors in space is demonstrated by the activities initiated by ESA and by national space agencies, CNES, ASI, DLR.

The study conducted by ESA on HYPER mission, proposed in 2000 in response to the call for the second and third Flexi-missions (F2/F3), showed the feasibility of cold atom interferometry in space both for inertial sensing and fundamental physics studies.

SAI, a new project funded by ESA, started in 2007 [20]. The project intends to exploit the potential of matter-wave sensors in microgravity for the measurement of acceleration, rotations, and faint forces. SAI aims to push present performances to the limits and to demonstrate this technology with a transportable sensor which will serve as a prototype for the space qualification of the final instrument. The atom interferometer will be used to perform fundamental physics tests and to develop applications in different areas of research (navigation, geodesy). These activities are financed by the HME Directorate in the frame of the ELIPS2 program. Several pieces of technology for this activity are common to those developed for other ESA projects based on cold atom clocks, namely, ACES (Atomic Clocks Ensemble in Space) and SOC (Space Optical Clocks).

Different proposals based on the utilization of matter-wave interferometers and atomic clocks for fundamental physics studies were submitted to ESA in the context of the "Cosmic Vision 2015-2025" program.

The applications of atomic quantum sensors in space are interdisciplinary, covering diverse and important topics. In fundamental physics, space-based cold atom sensors may be the key for new experiments, e.g., accurate tests of general relativity, search for new forces, test of $1 / \mathrm{r}^{2}$ law for gravitational force at micrometric distances, neutrality of atoms. Possible applications can be envisaged in astronomy and space navigation (inertial and angular references), realization of SI-units (definition of $\mathrm{kg}$, measurements of Newtonian gravitational constant $\mathrm{G}, \mathrm{h} / \mathrm{m}$ measurement), GALILEO and LISA technology, prospecting for resources and major Earth-science themes.

\section{Acknowledgments}

For the experiment on $G$, G.M.T. acknowledges seminal discussions with M.A. Kasevich and J. Faller and useful suggestions by A. Peters. M. Fattori, T. Petelski, and J. Stuhler contributed to setting up the apparatus. This work was supported by INFN (MAGIA experiment), EU (contract RII3CT-2003-506350 and FINAQS STREP/NEST project), INGV, ESA, ASI. 


\section{References}

1. A. Peters, K. Y. Chung and S. Chu, Nature 400, p. 849 (1999).

2. J. M. McGuirk, G. T. Foster, J. B. Fixler, M. J. Snadden and M. A. Kasevich, Phys. Rev. A 65, p. 033608 (2002).

3. A. Bertoldi, G. Lamporesi, L. Cacciapuoti, M. D. Angelis, M. Fattori, T. Petelski, A. Peters, M. Prevedelli, J. Stuhler and G. M. Tino, Eur. Phys. J. D 40, p. 271 (2006).

4. J. B. Fixler, G. T. Foster, J. M. McGuirk and M. Kasevich, Science 315, p. 74 (2007).

5. S. Fray, C. A. Diez, T. W. Haensch and M. Weitz, Phys. Rev. Lett. 93, p. 240404 (2004)

6. S. Dimopoulos, P. Graham, J. Hogan and M. Kasevich, Phys. Rev. Lett. 98, p. 111102 (2007).

7. G.M. Tino, in 2001: A Relativistic Spacetime Odyssey - Proceedings of JH Workshop, Firenze, 2001 (I. Ciufolini, D. Dominici, L. Lusanna eds., World Scientific, 2003). Also, Tino G. M., Nucl. Phys. B 113, 289 (2003).

8. S. Dimopoulos and A. A. Geraci, Phys. Rev. D 68, p. 124021 (2003).

9. D. M. Harber, J. M. Obrecht, J. M. McGuirk and E. A. Cornell, Phys. Rev. A 72, p. 033610 (2005).

10. G. Ferrari, N. Poli, F. Sorrentino and G. M. Tino, Phys. Rev. Lett. 97, p. 060402 (2006).

11. G. M. Tino, L. Cacciapuoti, K. Bongs, C. J. Bordé, P. Bouyer, H. Dittus, W. Ertmer, A. Gorlitz, M. Inguscio, A. Landragin, P. Lemonde, C. Laemmerzahl, A. Peters, E. Rasel, J. Reichel, C. Salomon, S. Schiller, W. Schleich, K. Sengstock, U. Sterr and M. Wilkens, Nucl. Phys. B (Proc. Suppl.) 166, p. 159 (2007).

12. G. Tino and F. Vetrano, Class. Quantum Grav. 24, 2167 (2007).

13. P. J. Mohr and B. N. Taylor, Rev. Mod. Phys. 77-1, 42 (2005).

14. M. Kasevich and S. Chu, Appl. Phys. B 54, p. 321 (1992).

15. G. Lamporesi, A. Bertoldi, A. Cecchetti, B. Dulach, M. Fattori, A. Malengo, S. Pettorruso, M. Prevedelli and G. Tino, Rev. Sci. Instrum. 78, p. 075109 (2007).

16. G. T. Foster, J. B. Fixler, J. M. McGuirk and M. A. Kasevich, Opt. Lett. 27, p. 951 (2002).

17. J. C. Long, H. W. Chan, A. B. Churnside, E. A. Gulbis, M. C. M. Varney and J. C. Price, Nature 421, p. 922 (2005).

18. S. J. Smullin, A. A. Geraci, D. M. Weld, J. Chiaverini, S. Holmes and A. Kapitulnik, Phys. Rev D 72, p. 122001 (2005).

19. P. Wolf, P. Lemonde, A. Lambrecht, S. Bize, A. Landragin and A. Clairon, Phys. Rev. A 75, p. 063608 (2007).

20. G. M. Tino et al., Space Atom Interferometers (SAI) (AO-2004-64), Proposal in response to ESA Announcement of Opportunity in Life and Physical Sciences and Applied Research Projects, ESA-AO-2004. 